\title{
Revisión sistemática del soporte nutricional en las úlceras por presión
}

\author{
D. DE LUIS, R. ALLER
}

\begin{abstract}
Sección de Endocrinología y Nutrición Clínica. Unidad de Apoyo a la Investigación. Hospital Universitario del Río Hortega. Instituto de Endocrinología y Nutrición Clínica. Facultad de Medicina. Valladolid
\end{abstract}

\section{RESUMEN}

Las úlceras por presión se definen como un área de daño localizada en la piel y en los tejidos subyacentes causado por la presión, fricción o combinación de ambos. La prevalencia de esta patología oscila entre un 3 a un $66 \%$ dependiendo del paciente y de la patología que presenta. La presencia de úlceras por presión se asocia con un incremento de la morbilidad y mortalidad. Dentro de los posibles factores de riesgo relacionados con las úlceras por presión, tenemos como uno de los más importantes la situación nutricional. Ante este tipo de pacientes podemos plantearnos realizar una intervención nutricional antes de que se desarrolle la úlcera (prevención primaria) o cuando la úlcera ya esta establecida (prevención secundaria). En el estudio de prevención primaria con mayor número de pacientes (662), los pacientes del grupo de intervención nutricional recibieron dos suplementos orales por día además de la dieta normal. El grupo control recibió la dieta hospitalaria estándar sola. La incidencia acumulada de úlceras por presión (todos los grados) fue del $40 \%$ $(118 / 295)$ en el grupo de intervención nutricional versus el $48 \%$ (181/377) en el grupo control. Esto equivale a un riesgo relativo de desarrollar un ulcera con la suplementación de 0,83 (IC95\%: 0,70 a 0,99). En los trabajos de prevención secundaria, al analizar los ensayos con nutrientes de manera individual, el zinc no ha demostrado su utilidad de manera aislada, y con respecto a la vitamina $\mathrm{C}$, obtenemos datos contradictorios en dos ensayos con la misma dosis de suplementación (500 mg cada 12 horas). En la actualidad los trabajos de intervención se realizan con suplementos nutricionales con diversos nutrientes (zinc, arginina, vitamina $\mathrm{C}$ ), habiendo demostrado algunos ensayos randomizados una mejoría de la cicatrización con estas fórmulas enriquecidas.

En la actualidad con los datos disponibles, parece que la suplementación nutricional con independencia de los micronutrientes empleados produce una disminución del riesgo de desarrrollar úlceras por presión en pacientes de riesgo. Sin embargo los estudios de prevención secundaria por su heterogeneidad no permiten obtener unas conclusiones claras. No obstante teniendo en cuenta los últimos trabajos, parece que los suplementos enriquecidos en arginina, zinc y vitamina C podría acelerar la cicatrización de estas úlceras.

PALABRAS CLAVE: Úlceras por presión. Soporte nutricional.
SYSTEMATIC REVIEW OF NUTRICIONAL SUPPORT IN PRESSURE ULCER

\section{ABSTRACT}

Pressure ulcer is an area of localised damage to the skin and underlying tissue caused by pressure, shear, friction and/or combination of these things. Prevalence of this entity is between 3 and 66\%, depending of the patients and the pathology. Pressure ulcer is associated with an increased risk of morbidity and mortality. One of the most important risk factors to develop a pressure ulcer is nutritional status. We can use different interventional strategies, first of all (primary intervention) before the patient has developed a ulcer and secondly, the treatment of a established ulcer (secondary prevention). In the most important primary prevention study with 662 patients, two oral nutritional supplements per day were given to the patients. The incidence of pressure ulcer was $40 \%$ $(118 / 295)$ in the interventional group and 48\% (181/377) in control group. A relative risk to develop a pressure ulcer with supplementation of 0.83 (C195\%: 0.70 a 0.99). In the studies with secondary prevention, when we analyze in an individual way the different nutrients, zinc has not demonstrated the utility in an independent way. Vitamin $C$ shows contradictory data in two randomized clinical trial with the same dose $(500 \mathrm{mg}$ each 12 hours). Recently, some randomized clinical trials have demonstrated an improvement in healing rates with enhanced enteral formulas (zinc, arginine, vitamin $C)$.

Oral supplementation without taking account micronutrients decreases risk of pressure ulcer. However, studies of secondary prevention due to heterogeneity have not let clear conclusions. However, enteral enhanced formula could improve ulcer healing.

KEY WORDS: Pressure ulcer. Nutritional support. 


\section{INTRODUCCIÓN}

Las úlceras por presión se definen como un área de daño localizada en la piel y en los tejidos subyacentes causado por la presión, fricción o combinación de ambos (1). La severidad de las úlceras permite clasificarlas en 4 grados desde el I al IV (Tabla I). La prevalencia de esta patología oscila entre un 3 a un $66 \%$ dependiendo del paciente y de la patología que presenta $(2,3)$.

\begin{tabular}{ll}
\multicolumn{1}{c}{ TABLA I } \\
\hline Estadio & \multicolumn{1}{c}{ Características } \\
\hline II & $\begin{array}{l}\text { Eritema en piel intacta que no cede con la presión } \\
\text { Afectación de la epidermis o dermis o de ambas. La úlcera es } \\
\text { III }\end{array}$ \\
IV & $\begin{array}{l}\text { Afecticiación de la piel en toda su profundidad con necrosis del teji- } \\
\text { do subcutáneo } \\
\text { Afectación en profundidad con destrucción y mecrosis de tejido } \\
\text { con afectación del músculo, hueso o estructuras adyacentes }\end{array}$ \\
\hline
\end{tabular}

La presencia de úlceras por presión se asocia con un incremento de la morbilidad y mortalidad. Existe entre 4 y 6 veces más riesgo de muerte en un paciente con úlcera por presión (4). La calidad de vida también se ve afectada, por el dolor, exudación y modificación de la imagen corporal (5). Las infecciones locales y generales son más prevalentes, necesitando más días de hospitalización (6). Por ello, las consecuencias económicas son enormes, el coste anual de tratar las úlceras por presión en Gran Bretaña se calcula en unos 1.065 millones de euros y en Estados Unidos de 2,4 billones de euros (6).

Dentro de los posibles factores de riesgo relacionados con las úlceras por presión, tenemos como uno de los más importantes la situación nutricional. Se ha implicado el bajo índice de masa corporal, el bajo peso, así como la reducción de la ingesta oral. Por tanto la mala situación nutricional aumenta el riesgo y empeora la evolución de las úlceras por presión (7). Los mecanismos por los que la situación nutricional está implicado en el riesgo y evolución de las úlceras por presión son múltiples. En primer lugar por la reducción del aporte de nutrientes al mantenimiento y reparación de los tejidos, en segundo lugar por la disminución de la masa grasa con su efecto almohadilla y en tercer lugar por la reducción de la actividad física, disminución de la motilidad y presencia de edema.

\section{EFECTOS DE DIFERENTES NUTRIENTES SOBRE LA CICATRIZACIÓN}

El correcto aporte de macro y micronutrientes es por tanto importante en esta patología. En principio es preciso un aporte hiperproteico que puede oscilar desde los 1,2 a 1,5 g de proteína $/ \mathrm{kg}$ de peso ideal, lo que representa aproximadamente un $20 \%$ de las necesidades calóricas. Durante los procesos de cicatrización, se incrementa la síntesis de proteínas en la zona de la herida. Si existe un déficit de aminoácidos, se reduce la síntesis de proteínas. Un ejemplo de esta situación es el aminoácido arginina, demostrándose que durante la cicatrización puede convertirse en un aminoácido esencial (8). Se ha demostrado que dosis de arginina entre 17 y $24 \mathrm{~g}$ mejoran el depósito de colágeno en las heridas (9).

Con respecto al aporte de hidratos de carbono, se recomiendan que representen entre un 55 y $60 \%$ del aporte calórico total y con un alto aporte de hidratos de carbono complejos. Los objetivos de este aporte son múltiples; en primer lugar aportar calorías a los pacientes para evitar el consumo de proteínas endógenas. En segundo lugar, la presencia de hidratos de carbono de absorción lenta permite que la glucemia no sea tan elevada y no se produzcan fenómenos de glicosilación proteica. Estos fenómenos de glicación pueden empeorar el trasporte de vitamina $\mathrm{C}$ al interior de las células $\mathrm{e}$ inhibir la proliferación de fibroblastos, produciendo un empeoramiento en la cicatrización de las heridas (10).

Las grasas, deben representar un 20-25\%, son importantes en los procesos de cicatrización al vehicular ácidos grasos esenciales como los ácidos linoleico y linolénico, componentes fundamentales de las membranas celulares, sobre todo en una situación de alto recambio tisular como es la cicatrización de una úlcera. Tampoco debemos olvidarnos que los ácidos grasos de la serie omega 3, presentan fenómenos antinflamatorios, inmunomoduladores y vasodilatadores, todos importantes para los procesos de cicatrización (11).

Las vitaminas y minerales, con los conocimientos fisiológicos que tenemos en la actualidad, existen una serie de ellas que presentan un gran interés (vitamina $\mathrm{C}$, vitamina $\mathrm{E}$, vitamina A, vitamina K, hierro, cobre y zinc). Probablemente la que más interés despierte es la vitamina $\mathrm{C}$. Esta vitamina es esencial como cofactor de la síntesis de colágeno y para mantener la función inmunológica. Las recomendaciones internacionales se sitúan en los sujetos sanos en 75 mg/día (mujeres) y $95 \mathrm{mg} /$ día (varones), con un límite tolerable de $2 \mathrm{~g}$ al día (12). La vitamina A promueve la diferenciación de los fibroblastos, la síntesis de colágeno y reduce las infecciones de las heridas (13). El papel de la vitamina E permanece como uno de los grandes interrogantes en la cicatrización, no estando claro la necesidad de su suplementación en este tipo de patologías (14). Con respecto a la vitamina $\mathrm{K}$ es necesaria como cofactor para la síntesis de factores de coagulación, siendo producida normalmente por las bacterias del intestino. Los pacientes que reciben antibioterapia, como puede ser el caso de ulceras por presión infectadas, van a disminuir la producción endógena de vitamina K precisando suplementación (14).

Respecto a los minerales, el cobre es un importante cofactor para muchos enzimas, incluyendo la lisil oxidasa, implicada en los enlaces cruzados del colágeno y de la elastina (15). El hierro es otro de los micronutrientes implicados en la hidroxilación de la prolina y lisina, necesarios para la síntesis de colágeno. Por otra parte la anemia por déficit de hierro va a producir una disminución de la oxigenación tisular, así como una reducción en la actividad bactericida de los leucocitos (14). Probablemente el mineral que más interés ha despertado en los procesos de cicatrización es el zinc. Este mineral implicado como coenzima en multitud de vías metabólicas y procesos de división celular, es necesario a altas concentraciones en el foco cicatricial, no obstante no esta exento de riesgos en cantidades excesivas, relacionandose con episodios de diarrea, alteraciones del sistema inmunológico y en el metabolismo del cobre (15). 
ANÁLISIS DE LOS DIFERENTES ESTUDIOS DE INTERVENCIÓN NUTRICIONAL

Ante este tipo de pacientes podemos plantearnos realizar una intervención nutricional antes de que se desarrolle la úlcera (prevención primaria) o cuando la úlcera ya esta establecida (prevención secundaria).

Con respecto a los estudios de prevención primaria, el primero fue realizado por Delmi y cols. (16). Se evaluaron un total de 59 pacientes en recuperación de fracturas de cadera, con un seguimiento de seis meses, los pacientes recibieron la dieta hospitalaria estándar sola o un suplemento nutricional oral diario además de la dieta hospitalaria. El número de úlceras por presión (de todos los grados) en el primer centro fue de dos en el grupo de tratamiento $(n=27 ; 7 \%)$ y de tres en el grupo de control $(n=32 ; 9 \%)$ con un riesgo relativo $(\mathrm{RR})$ estadísticamente no significativo para las úlceras por presión de 0,79 . El número de úlceras por presión en el segundo centro (recuperación) fue de cero en el grupo de tratamiento $(\mathrm{n}=$ 9) y de tres en el grupo control $(n=15 ; 20 \%)$ con un RR que no fue estadísticamente significativo de 0,23 . El número de úlceras por presión presente a los seis meses fue de cero en el grupo de tratamiento $(\mathrm{n}=25)$ y de dos en el grupo de control $(\mathrm{n}=27 ; 7,4 \%)$ con un RR que no fue estadísticamente significativo de 0,22 .

En el siguiente estudio realizado por el grupo de Hartgrink y cols. (17), se evaluaron a 140 pacientes en recuperación de fracturas de cadera durante 2 semanas, el grupo de intervención fue tratado con la dieta hospitalaria estándar y alimentación adicional por sonda nasogástrica que se administró con una bomba de alimentación durante la noche. El grupo control recibió la dieta hospitalaria estándar sola. Un total de 25 de los 48 pacientes del grupo de intervención (52\%) y 30 de los 53 pacientes del grupo de control $(56 \%)$ tuvieron úlceras por presión de grado 2 o más, sin detectar diferencias significativas.

Sin embargo el estudio con mayor número de pacientes ha sido realizado por Bourdel y cols. (18), mostrando unos resultados muy diferentes a los anteriores. Los pacientes del grupo de intervención nutricional recibieron dos suplementos orales por día además de la dieta normal. El grupo control recibió la dieta hospitalaria estándar sola. Se reclutaron un total de 662 pacientes mayores de 65 años, en la fase aguda de una enfermedad crítica, con un seguimiento hasta el alta hospitalaria. La incidencia acumulada de úlceras por presión (todos los grados) fue del 40\% (118/295) en el grupo de intervención nutricional versus el $48 \%$ (181/377) en el grupo de control. Esto equivale a un riesgo relativo de desarrollar un ulcera con la suplementación de 0,83 (IC95\%: 0,70 a 0,99). El análisis multivariado mostró como los factores de riesgo independientes de desarrollar una úlcera por presión fueron: nivel de albúmina sérica, puntuación de Kuntzman, fractura de miembro inferior, puntuación de Norton $<10$ y pertenencia al grupo de control.

Houwing y cols. (2003) (19) realizaron el seguimiento de 103 pacientes con fractura de cadera durante 1 mes. El grupo de intervención recibió un suplemento diario además de la dieta hospitalaria estándar. El grupo de comparación recibió la dieta hospitalaria estándar y un placebo a base de una bebida sin calorías. Después de dos semanas, la incidencia de úlceras por presión (estadio I-II) fue de 55\% $(27,51)$ en el grupo de intervención nutricional y $59 \%$ (30/52) en el grupo de placebo, sin alcanzar la significación estadística. La inciden- cia de úlceras por presión en estadio II fue de $18 \%$ en el grupo de intervención nutricional versus $28 \%$ en el grupo de placebo, sin significación estadística.

Evaluando la literatura tenemos el segundo bloque de trabajos en prevención secundaria, con ulceras de presión instauradas. En este caso el análisis de los trabajos lo vamos a realizar teniendo en cuenta el nutriente suplementado. Con respecto al zinc, Norris y cols. (20), trataron un total de 10 pacientes con sulfato de zinc, los pacientes recibieron 3 x 200 $\mathrm{mg}$ de sulfato de zinc por día o placebo durante un período de 24 semanas. Los pacientes tratados con zinc con un cambio en el volumen de la úlcera por presión de $10 \mathrm{ml}$ frente a 10 pacientes que recibieron placebo con un cambio en volumen de $6,0 \mathrm{ml}$, sin alcanzar la significación estadística.

Otro de los nutrientes estudiados ha sido la vitamina C. De este modo Taylor y cols. (21), evaluaron un total de 20 pacientes. En el grupo tratado con ácido ascórbico $(500 \mathrm{mg}$ dos veces al día, durante 4 semanas) hubo una reducción promedio estadísticamente significativa en el área de úlcera por presión del $84 \%$ en comparación con el $42,7 \%$ en el grupo de placebo, (IC95\%: 34,72 a 47,88 p <0,005). La tasa de cicatrización promedio fue de $2,47 \mathrm{~cm}^{2} /$ semana en el grupo de intervención comparada con $1,45 \mathrm{~cm}^{2} / \mathrm{semana}$ en el grupo de control. Otro trabajo realizado posteriormente con vitamina $\mathrm{C}$ (22), comparando $500 \mathrm{mg}$ dos veces al dia frente a $10 \mathrm{mg} / \mathrm{dos}$ veces al día durante 12 semanas mostró datos sorprendentes. La tasa de cicatrización absoluta promedio en el grupo de intervención $(\mathrm{n}=43)$ fue de $0,21 \mathrm{~cm}^{2} /$ semana y de 0,27 $\mathrm{cm}^{2} /$ semana en el grupo control $(\mathrm{n}=45)$, sin diferencias significativas. La reducción del volumen promedio fue de 0 $\mathrm{ml} / \mathrm{semana}$ en el grupo de intervención y de $0,20 \mathrm{ml} / \mathrm{semana}$ en el grupo control (diferencia: -0,20 ml/semana). El "cambio clínico" promedio donde las mejorías (es decir, reducción de la superficie, velocidad de la cicatrización, reducción del volumen) fue de 17,89\%/semana en el grupo de intervención y $26,08 \% /$ semana en el grupo de control.

Con respecto a la suplementación con proteínas, el trabajo de Chernoff y cols. (23) con una fórmula nutricional de alto contenido proteico o de muy alto contenido proteico y se monitorizaron durante ocho semanas. Aunque el tamaño de las úlceras se redujo con ambas dietas, la mejoría fue mayor en el grupo de alto contenido proteico. Ninguno de los pacientes del grupo de alto contenido proteico y cuatro pacientes del grupo de muy alto contenido proteico tuvieron una cicatrización completa de su úlcera, sin diferencias estadisticamente significativas.

La reducción promedio del tamaño de la úlcera fue del $42 \%$ en el grupo de alto contenido proteico en comparación con el $73 \%$ en el grupo de muy alto contenido proteico.

Recientemente han aparecido dos trabajos que utilizan en prevención secundarias dos suplementos nutricionales enriquecidos con varios de los nutrientes que hemos analizado anteriormente (proteínas, arginina, vitamina C) y también arginina. En el trabajo de Frías Soriano y cols. (23) se trataron 39 pacientes con ulceras de presión estadio III y IV, en una sola rama de intervención con un suplemento que contenía 3 $\mathrm{g}$ de arginina, $250 \mathrm{mg}$ de vitamina C, y $9 \mathrm{mg}$ de zinc, mostrando una reducción del $29 \%$ del tamaño de la ulcera, con una reducción significativa de la exudación y del tejido necrótico. En el trabajo de Desneves (24), se randomizaron 16 pacientes con úlceras por presión con estadios II, III y IV en cada grupo, recibiendo el primer grupo una dieta estándar del Hospital, el 
segundo grupo una dieta estándar junto a un suplemento hiperproteico y energético y el tercer grupo la dieta estándar junto aun suplemento hiperproteico y energético enriquecido en arginina $9 \mathrm{~g}$, vitamina C $500 \mathrm{mg}$ y zinc $30 \mathrm{mg}$. Mostrando solo mejoría estadísticamente significativa en los procesos de cicatrización de la úlcera el grupo con el preparado enriquecido (puntuación PUSH (Pressure Ulcer Scale for Healing) $(9,4$ $+1,2$ vs. 2,6 + 0,6 puntos).

Recientemente ha aparecido un metanálisis (25), en el cual se evalúan 15 trabajos de los cuales 8 son ensayos randomizados, donde se demuestra como los suplemento orales (250-500 kcal) durante 2-26 semanas se asocian con un incidencia baja de desarrollar ulceras por presión $(0,75$; IC95\% 0,62-0,89) en pacientes de alto riesgo. Este trabajo con respecto a los estudios de prevención secundaria, señala que dado la heterogeneidad de los suplementos utilizados solo se puede comentar que analizados individualmente los diferentes diseños, los preparados enriquecidos en algunos nutrientes parecen ser superiores los preparados estándar.

En la actualidad las perspectivas en el tratamiento nutricional de esta patología son muy interesantes. Por ejemplo, el grupo de Lee y cols. (26) han demostrado en un ensayo clíni- co aumentar la tasa de cicatrización de ulceras por presión (II,II y IV) con una fórmula enriquecida en hidrolizado de proteínas procedentes de colágeno. También es necesario recordar que todos estos conocimientos sobre la cicatrización en úlceras por presión pueden ser aplicados en otros modelos de heridas, como son las heridas quirúrgicas $(27,28)$.

\section{CONCLUSIONES}

La úlcera por presión es una patología frecuente que genera un claro aumento de la morbimortalidad. Los estudios de prevención primaria y secundaria son necesarios. En la actualidad con los datos disponibles, parece que la suplementación nutricional con independencia de los micronutrientes empleados produce una disminución del riesgo de desarrrollar úlceras por presión en pacientes de riesgo. Sin embargo los estudios de prevención secundaria por su heterogeneidad no permiten obtener unas conclusiones claras. No obstante teniendo en cuenta los últimos trabajos, parece que los suplementos enriquecidos en arginina, zinc y vitamina $\mathrm{C}$ podría acelerar la cicatrización de estas úlceras.

\section{Bibliografía}

1. EDUAP (European Pressure Ulcer Advisory Panel), 2004. Pressure Ulcer Treatment Guidelines. http//www .epuap.org/gltreatment .html.

2. Tannen A, Dassen T, Bours G, Halfens R. A comparison of pressure ulcer prevalence: Concerted data collection in the Netherlands and Germany. Int J Nurs Stud 2004; 41: 607-12.

3. Whitfield M, Kaltenhaler E, Akehurst R, Walters S, Paisley S. How effective are prevention strategies in reducing the prevalence of pressure ulcers? J Wound Care 2000; 9: 261-6.

4. Allman RM, Laprade CA, Noel LB, Walker JM, Moorer CA, Dear MR, et al. Pressure sores among hospitalized patients. Ann Int Med 1986; 105: 337-42

5. Fox C. Living with a pressure ulcer: a descriptive study of patients experiences. Br J Community Nurs Wound Care 2002; S10: 12-4.

6. Sttraton RJ, Green CJ, Elia M. Consequences of diease related malnutrition. In: stratton rj, green cj, elia . Disease-related malnutrition: An evidence based approach to treatment. CABI Publishing. Wallingford, Oxon UK. p. 113-55.

7. Arris CL, Fraser C. Malnutrition in the institutionalized elderly: The effects on woung healing. Ostomy Wound Manage 2004; 50: 54-63.

8. de Luis DA, Aller R, Izaola O. Arginina: Un viejo amigo, a debate. Formacion Continuada Metabolismo y Nutrición 2005; 1: 40-5.

9. Barbul A, Lazarou SA, Efron DT. Arginine enhances wound healing and lymphocytes immune responses in humans. Surgery 1990; 108: 331-7.

10. Hehenberger K, Heilborn JD, Brismar K. Inhibited oroliferation of fibroblasts derived from chronic diabetic wounds and normal dermal fibroblasts treated with high glucose is associated with increased formation of 1-lactate. Wound Repair Regen 1998; 6: 135-41.

11. Mayes T, Gottschlich MM. Burns, Wound Healing. In: The science and practice of nutrition support: A case based core currículo. Kendall/Hunt Publishing Co. Iowa; 2001. p. 391-420.

12. Monsen E. Dietary reference intakes for the antioxidant nutrients: Vitamin C, selenium and carotenoids. J Am Diet 2000;100:637-640.

13. Cohen IK, Diegelmann RF, Lindblad WJ. Wound healing: Biochemicalo and clinical aspects. W.B. Saunders Co. Toronto; 1992. p. 248-73.

14. Baumann LS, Spencer J. The effects of topical vitamin E on the cosmetic appearance of scars. Dermatol Surg 1999; 25: 311-5.

15. Trumbo P, Yates A, Schlicken S. Dietary reference intakes: Vitamin A, vitamin $\mathrm{K}$, arsenic, boron, chomium, copper, iodine, iron, manganese, molylodenum, nickel, silicom, vanadium, zinc. J AM Diet Assoc 2001; 101: 294-301.

16. Delmi M, Rapin CH, Bengoa JM, Delmas PD, Vasey H, Bonjour JP. Dietary supplementation in elderly patients with fractured neck of the femur. Lancet 1990; 335: 1013-6.

17. Hartgrink HH, Wille J, Konig P, Hermans J, Breslau PJ. Pressure sores and tube feeding in patients with a fracture of the hip: A randomized clinical trial. Clin Nutr 1998; 17: 287-92.

18. Bourdel-Marchasson I, Barateau M, Rondeau V, Dequae-Merchadou L, Salles-Montaudon N, Emeriau JP, et al. A multi-center trial of the effects of oral nutritional supplementation in critically ill older inpatients. GAGE Group. Groupe Aquitain Geriatrique d'Evaluation. Nutrition 2000; 16: 1-5.

19. Houwing R, Rozendaal M, Wouters-Wesseling W, Beulens JWJ, Buskens E, Haalboom J. A randomised, double-blind assessment of the effect of nutritional supplementation on the prevention of pressure ulcers in hip-fracture patients. Clinical Nutrition 2003; 22: 401-5.

20. Norris JR, Reynolds RE. The effect of oral zinc sulfate therapy on decubitus ulcers. J Am Geriatr Soc 1971; 19: 793-7.

21. Taylor TV, Rimmer S, Day B, Butcher J, Dymock IW. Ascorbic acid supplementation in the treatment of pressure-sores. Lancet 1974; 7: 2: 544-6.

22. ter Riet G, Kessels AG, Knipschild PG. Randomized clinical trial of ascorbic acid in the treatment of pressure ulcers. J Clin Epidemiol 1995; 48: 1453-60.

23. Frías Soriano L, Lage Vázquez MA, Pérez Portabella C, Xandri Graupera JM, Wouters Wesseling, Wagenaar L. The effectivenes of ora nutricional supplementation in the healing of pressure ulcers. J Wound Care 2004; 13: 319-23

24. Desneves KJ, Todorovic BE, Cassar A, Crowe TC. Tretment with supplementary arginine, vitamin $\mathrm{C}$ and zinc in patients with pressure ulcers: A randomized controlled trial. Clin Nutr 2005: 24: 979-87.

25. Stratton R, Ek AC, Engfer M, Moore Z, Rigby P, Wolfe R, Elia M. Enteral nutrition support in prevention and treatment of pressure ulcers: A systematic review and meta-analysis. Ageing Research Reviews 2005; 4: 422-50

26. Lee SK, Posthauer ME, Dorner B, Redovian V, Maloney MJ. Pressure ulcer healing with concentrated, fotified, collagen protein hydrolysate supplement: A randomized controlled trial. Adv Skin Wound Care 2006; 19: 92-6

27. De Luis DA, Izaola O, Aller R, Cuellar L, Terroba MC. Postsurgery enteral immunonutrition in head and neck cancer patients. Eur J Clin Nutr 2002; 56: 1126-9

28. De Luis DA, Arranz M, Aller R, Izaola O, Cuellar L, Terroba MC. Immuonenhanced enteral nutrition, effect on inflammatory markers in head and neck cancer patients. Eur J Clin Nutr 2005; 1-4. 